Surface Modified Electrospun Porous Magnetic Hollow Fibers Using Secondary Downstream Collection

\title{
Solvent Contouring
}

Shuting Wu ${ }^{\mathrm{a}, \mathrm{b}}$, Baolin Wanga,b, Zeeshan Ahmad ${ }^{\mathrm{c}}$, Jie Huang ${ }^{\mathrm{d}}$, Ming-Wei Changa,b*, Jing-Song Li ${ }^{\mathrm{a}}$

${ }^{a}$ Key Laboratory for Biomedical Engineering of Education Ministry of China, Hangzhou, 310027, P. R. China.

${ }^{\mathrm{b}}$ Zhejiang Provincial Key Laboratory of Cardio-Cerebral Vascular Detection Technology and Medicinal

Effectiveness Appraisal, Zhejiang University, Hangzhou, 310027, P.R. China.

'Leicester School of Pharmacy, De Montfort, University, The Gateway, Leicester, LE1 9BH, UK.

${ }^{\mathrm{d}}$ Department of Mechanical Engineering, University College London, London, WC1E 7JE, UK. 


\begin{abstract}
In this study, a relatively facile porous magnetic hollow fiber engineering electrospinning method is demonstrated, which modulates fiber morphology based on secondary solvents (at variable temperatures). To demonstrate this, polycaprolactone (PCL) polymer and iron oxide nanoparticles (NPs) were used as the fibrous composite matrix. Fiber pore size increased with increasing immersed secondary solvent temperature. By contouring the surface morphology (via modulation of secondary collection solvents) of hollow magnetic fibers, drug (ketoconazole) release kinetics from spun mats were tuned. Furthermore, applying an external AC magnetic field to NP embedded porous fibers enhanced drug release. These findings are promising for altenative engineering, tuning and controlling fiber morphology and drug release behavior.
\end{abstract}

Keywords:Polymers; Porous Fiber; Surface morphology; electrospinning; coaxial; enhanced drug release.

\title{
1. Introduction
}

Electrospinning (ES) is widely used to engineer continuous micro-meter and nano-meter scaled fibers from a host of materials for a variety of healthcare applications[1,2]. Based on selected materials, process optimization (e.g. flow rate, applied voltage and working distance) and media parameters (e.g. viscosity and electrical conductivity), fibers with desirable properties are generated[2]. Coaxial ES (COES) has been used to engineer core-shell fibers with large surface to volume ratios to be effective for controlled drug release and tissue engineering [3]. However, modulation of fiber surface morphology remains limited.

Polycaprolactone (PCL) is a biocompatibile polymer, demonstrated by extensive biomateral explorations[4]. However, PCL drug carriers exhibit slow release rates due to the hydrophilic nature of polymers [3]. In order to achieve therapeutically ideal concentrations of released active, several excipients are embedded within PCL polymer matrix [5]. Also, hollow fibers possess super specific surface areas (compared to solid fibers); permitting facilitated drug release from inner and outer faces[6]. In addtion, magnetic NPs (MNPs) are innovative functional materials which have been used to enable an enhanced release of the active ingredient [7]. However, challenges remain for the generation of hollow fibers with varied surface morphology for drug delivery applications.

In this study, a facile approach for porous magnetic hollow fiber production with fine tuned surface morphology is demonstrated. The role of secondary downstream solvent substrates (treating fibers with a second solvent (and temperature) after collecting fibers in a primary solvent) are explored as potential routes to modulate morphology and surface features. Pore formation was explored using various secondary downstream solvents 
(at selected temperatures). Model drug release from porous magnetic hollow fibers was studied (using ambient and externally stimulated environments).

\section{Materials and methods}

\subsection{Materials}

Polycaprolactone (PCL, $\mathrm{M}_{w}=8 \times 10^{4} \mathrm{~g} / \mathrm{mol}$ ) was obtained from Sigma-Aldrich, USA. Ketoconazole (KCZ, 99.7\%) was purchased from ZhongtianInstrument Chemical, China. Magnetic nanoparticles $\left(\mathrm{Fe}_{3} \mathrm{O}_{4}, \mathrm{MNPs}\right.$, mean size: 20nm) were provided by HWRK Chem, China. Dichloromethane (DCM) and PBS were purchased from Sinopharm Chemical Reagent, China. Collecting solvents, including n-butanol, methanol, tetraethyl orthosilicate, 1,2-propanediol, ethanol and dimethyl silicone oil were obtained from Aladdin chemistry, China. All chemicals were of the analytic grade. PCL (10wt.\%) and KCZ (0.5wt.\%) were dissolved in DCM for 2 hours using magnetic stirrer (VELP, Italy). MNPs (0.5wt.\%) were added to the solution and the mixture was dispersed using bath sonication (2 hours). The final solution was used as shell material (for COES) while dimethyl silicon oil (as obtained) was the core material.

\subsection{Fiber Fabrication using secondary collection solvent}

An experimental COES set-up was used to produce porous magnetic hollow fibers (Fig.S1). The two COES media (suspension and oil) were simultaneously infused into the nozzle (inner dimethyl silicone oil=0.3ml/h and outer composite suspension $=9.8 \mathrm{ml} / \mathrm{h})$ under influence of an electric field $(\sim 18 \mathrm{kV})$. Once COES stable Taylor cone was achieved, homogeneous fibers were deposited on a glass slide coated with primary collecting medium (distance $=18 \mathrm{~cm}$ ) for $15 \mathrm{~s}$. Immediately after deposition, fiber coated glass substrate was immersed into secondary downstream selected solvent environment (1min) to ensure interaction. In order to examine solvent temperature impact on fiber morphology, fibers were immersed in ethanol using secondary solvents at 9, 20 and $38^{\circ} \mathrm{C}$. Fibers were desiccated (5days) after which dry porous magnetic hollow structures were analysed and tested further.

\subsection{Characterization}

Fiber morphology was assessed using optical (OM,Phenix BMC503-ICCF, China) and scanning electron microscopy (SEM,ProX, Phenom, Eindhoven, Netherlands). Fibers were analyzed using Fourier Transform infrared spectroscopy (FTIR, IR Affinity1, Shimadzu, Japan). Spectra were recorded from 400 to $4800 \mathrm{~cm}^{-1}$ $\left(\right.$ resolution $\left.=4 \mathrm{~cm}^{-1}\right)$. Water contact angle measurements on fibrous mats were performed using an optical contact angle and interface tension meter (SL200KB, KINO Industry Ltd.,USA). Fiber diameter and pore size 
were measured using ImageJ software (National Institute of Health,USA). Mean diameter was obtained using 100 randomly selected fibers for each sample. The mean pore size was quantified using 40 measurements for each sample.

\subsection{In vitro drug release study}

Each sample analyzed for in vitro drug release comprised 30mg of fiber mats and 10mL PBS (pH=7.4) using a HZ-8801K thermostatic oscillator (TSEFactory, China) over 192hrs. Moreover, the effect of an external AC magnetic field $(1.5 \mathrm{mT})$ on drug release characteristics was compared to non-triggered counterpart. The characteristic UV absorbance peak of KCZ in PBS was 202nm (UV-2600 spectrophotometer, Shimadzu, Japan)[3]. KCZ release was obtained using triplicate samples. The encapsulation efficiency (EE) was obtained using Equation 1[8]:

$\mathrm{EE}(\%)=$ Amount of drug encapsulated in fibers/Theoretical total amount of drug $\times 100 \%$ (1)

\section{Results and discussion}

Porous features can be afforded through non-solvent induced phase separation [9]. Ideal experimental conditions were obtained through optimization (applied voltage: $18 \mathrm{kV}$, collector distance: $17 \mathrm{~cm}$, shell/core flow rate:9.8/0.3 $\mathrm{mLh}^{-1}$ ), and stable processing of co-flowing media (electrospun fibers). Homogeneous fibers were deposited on to glass substrates coated with pre-selected primary solvents (i.e. n-butanol, methanol, tetraethyl orthosilicate, 1,2-propanediol and ethanol). For identification purposes in this study, resulting fibers are abbreviated to $F_{\text {but }}, F_{\text {met }}, F_{\text {teos }}, F_{\text {pro }}$ and $F_{\text {eth }}$, respectively. Fig. $S 2$ shows optical micrographs of electrospun fibers; $F_{\text {but }}, F_{\text {met }}, F_{\text {teos }}, F_{\text {pro }}$ and $F_{\text {eth. }}$ The results indicate fibers collected in various media were near uniform displaying mean diameters of $15.8,14.3,13.7,14.8$ and $13.1 \mu \mathrm{m}$ for $F_{\text {but }}, F_{\text {met }}, F_{\text {teos }}, F_{\text {pro }}$ and $F_{\text {eth }}$, respectively. Fig. 1 shows electron micrographs of generated fibers. The surface morphology of all mats appear porous with a rough surface topography and numerous small cavities are evident. Pore formation and structure can be explained due to an amalgamation of medium properties (i.e. molecular weight, viscosity and functional groups). The physical properties of collecting solvents are shown in Table $\mathrm{S} 1 . \mathrm{F}_{\text {but }}$ and $\mathrm{F}_{\text {met }}$ possess large surface pores, which is ascribed to low viscosity of their respective solvents [9] and such porous morphology are desirable for applications in tissue engineering, which improves cell adhesion, proliferation, and cell growth $[10]$. 


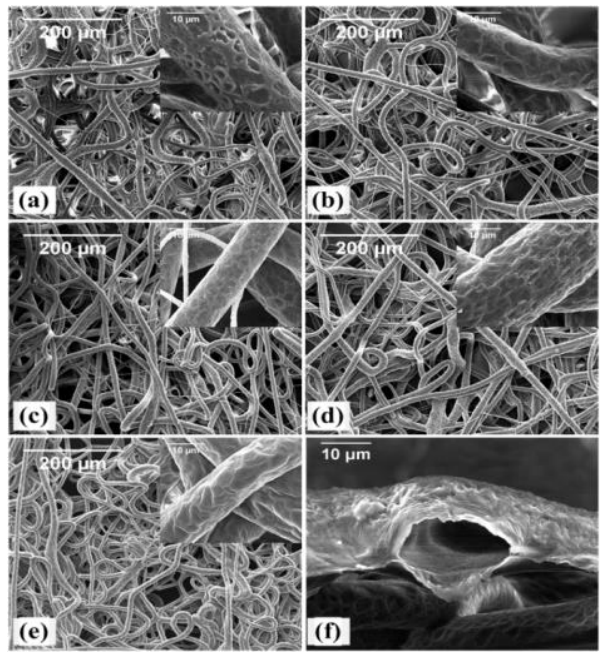

Figure1. Electron micrographs of coaxial electrospun porous hollow fibers collected in different solvents:(a) n-butanol, (b) methanol, (c) tetraethyl orthosilicate, (d) 1,2-propanediol and (e)ethanol, (f)hollow fibers collected in ethanol.

In order to investigate secondary downstream collection solvent temperature effect on fiber morphology, ethanol was selected as the second medium due to its economic cost, low toxicity, safety and access to previous experimental data. The collecting temperature was set to 9,20 and $38^{\circ} \mathrm{C}$ (denoted herein as $\mathrm{F}_{9}, \mathrm{~F}_{20}$ and $\mathrm{F}_{38}$, respectively). During experimental process, all other parameters were kept constant. Fig. 2 show fiber images of $\mathrm{F}_{9}, \mathrm{~F}_{20}$ and $\mathrm{F}_{38}$, respectively. Electron micrographs indicate increasing the solvent temperature gives rise to a topographical transition from distinctively defined (Fig. 2a', mean pore size $2.2 \mu \mathrm{m}$, mean fiber diameter $15.8 \mu \mathrm{m}$ ) to broad shallow pores (Fig. 2c', mean pore size $\sim 3.8 \mu \mathrm{m}$, mean fiber diameter $\sim 15.6 \mu \mathrm{m}$ ). The change in fiber pore can be explained with diffusion. As the collecting solvent temperature increases, the molecular motion of solvent becomes vigorous. Thus, the diffusion rate of solvent molecules in contact with solidifying fibers increases and a greater area of the fiber is affected (i.e. pore formation area). Varying the solvent temperature provides a facile and efficient method to refine and contour fiber morphology. 


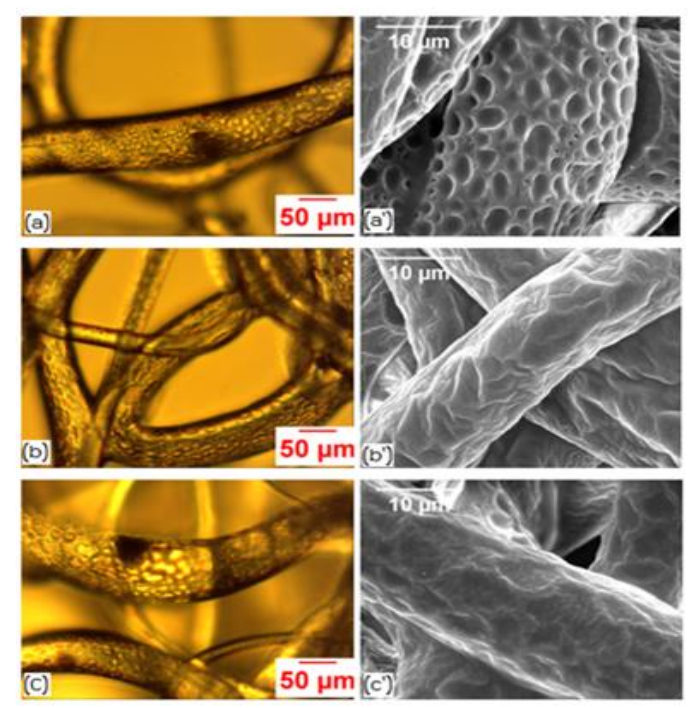

Figure 2. Optical micrograph of coaxial electrospun porous hollow fibers immersed in ethanol solvent at (a)

9, (b) 20 , (c) $38^{\circ} \mathrm{C},\left(\mathrm{a}^{\prime}-\mathrm{c}^{\prime}\right)$ electron micrographs of corresponding fibers shown in (a-c), respectively.

Fig. 3a shows a porous fibrous magnetic hollow mat responding to an external magnetic source. Through the action of a permanent magnetic source, relocation and aligment in the vial is observed. The magnetic sensitivity of fibrous mats to a suitable external source can therefore be managed (location and targetting) indicating in vivo potemtial.

Fig. 3 b shows drug release data from samples prepared using ethanol collection solvent at 9,20 and $38^{\circ} \mathrm{C}$. The cumulative release profiles from fibers deposited in ethanol solvent at all temperatures were similar to that of fiber system collected without the solvent. A typical biphasic release pattern was observed, namely a burst release followed by a slow sustained release, reaching $90 \%$ after $144 \mathrm{~h}$. However, these results can be explained based on the structure of hollow porous fibers. Unlike a solid matrix filament (or tube) a hollow structure possesses a larger surface to volume ratio which enables the release of drug from two faces (inner and outer). In addition, hollow structures demonstrate expedited drug release rates when compared to their smooth solid counterparts, due to the presence of abundant space/pores providing substantial media interaction [8]. Fig. 3c shows enhanced release from porous hollow fibers $\left(\mathrm{F}_{20}\right)$ under an external AC magnetic field (AMF, 40kHz). An external magnetic trigger (surrounding fibrous hollow mat in PBS) enhanced $\mathrm{KCZ}$ release, when compared to non-treated samples. The percentage of triggered KCZ release from porous magnetic fibers reached $>90 \%$ after 480 min. In comparison, only $~ 50 \%$ of $\mathrm{KCZ}$ was released during the same time from non-triggered samples. This is attributed to an increase in molecular motion within the polymeric matrix via MNP responding to the trigger, which may impact PCL polymer integrity. 
(a)

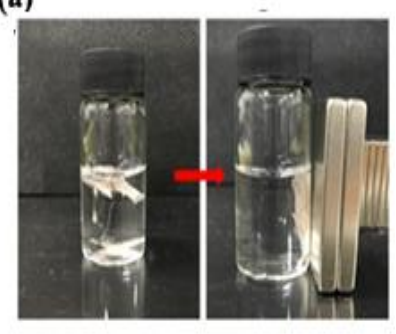

(b)

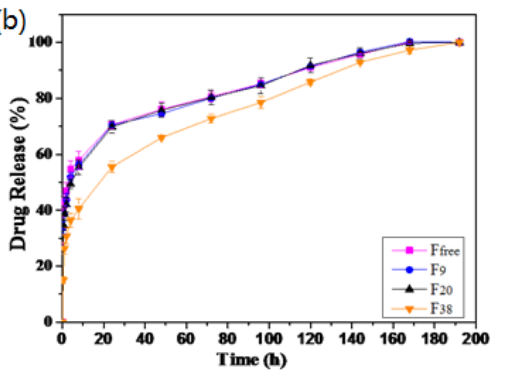

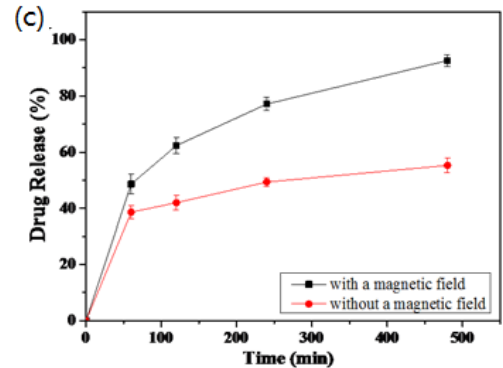

Figure 3. (a) Fibrous mat response to an externally applied magnetic source, (b) In vitro release profiles of KCZ-loaded fibers collected in 9, 20 and $38^{\circ} \mathrm{C}$ ethanol, respectively, (c) In vitro release profiles of magnetic porous hollow fibers $\left(\mathrm{F}_{20}\right)$ with and without an externally applied magnetic field.

\section{Conclusions}

Fiber morphology control, especially on the micron-scale, has recieved extensive attention. The contouring of electrospun fibers with incremental changes to topography remains a challenge and is essential to develop greater understanding of bio-material interfaces. In this study, an effective method to engineer magnetic hollow porous PCL fibers with control on micron scale fiber surface enhancment is demonstrated using secondary collection solvents and environments. The results indicate collecting solvent substrate type and temperature has a significant effect on the morphology of porous hollow fibers. Fibers can also be prepared with varying surface morphology, through pore tailoring, which can be designed to meet the requirements for modulated drug release and enhanced by an external $\mathrm{AC}$ magnetic field when required.

\section{Acknowledgements}

This work was financially supported by the Key Technologies R\&D Program of Zhejiang Province (2015C02035) and the Fundamental Research Funds for the Central Universities(2017QNA5017) .

\section{References}

[1] Fu R, Chen S, Lin Y, Zhang S, Jiang J, Li Q, et al. Improved Piezoelectric Properties of Electrospun Poly(vinylidene fluoride) Fibers Blended with Cellulose Nanocrystals. Mater Lett. 2016;187:86-8.

[2] Wu K, Yang W, Liu X, Jiao Y, Zhou C. Electrospun porous polyethersulfone (PES) fiber mats with high bilirubin adsorption capacity. Mater Lett. 2016;185:252-5.

[3] Wang B, Zheng H, Chang MW, Ahmad Z, Li JS. Hollow polycaprolactone composite fibers for controlled magnetic responsive antifungal drug release. Colloid Surface B. 2016;145:757-67.

[4] Xie J, Zhong S, Ma B, Shuler FD, Lim CT. Controlled biomineralization of electrospun poly( $\varepsilon$ caprolactone) fibers to enhance their mechanical properties. Acta Biomater. 2013;9:5698-707.

[5] Tiwari AP, Joshi MK, Kim JI, Unnithan AR, Lee J, Chan HP, et al. Bimodal fibrous structures for tissue engineering: Fabrication, characterization and in vitro biocompatibility. J Colloid Interface Sci. 2016;476:2934. 
[6] Song F, Shen X, Liu M, Xiang J. Formation and characterization of magnetic barium ferrite hollow fibers with high specific surface area via sol-gel process. Solid State Sci. 2010;12:1603-7.

[7] Huang SR, Lin KF, Don TM, Chiu WY, Lin MF. Fabrication and characterization of UV-crosslinkable thermoresponsive composite fibers with magnetic properties. J Polym Sci Pol Chem. 2015;53:2152-62.

[8] Gao Y, Chang MW, Ahmad Z, Li JS. Magnetic-responsive microparticles with customized porosity for drug delivery. RSc Adv. 2016;6.

[9] Gao J, Li W, Wong SP, Hu M, Li RKY. Controllable morphology and wettability of polymer microspheres prepared by nonsolvent assisted electrospraying. Polymer. 2014;55:2913-20.

[10] Yang A, Huang Z, Yin G, Pu X. Fabrication of aligned, porous and conductive fibers and their effects on cell adhesion and guidance. Colloid Surface B. 2015;134:469-74. 